\title{
Geoestatística como ferramenta para estudos da variabilidade da precipitação pluviométrica no estado de Pernambuco, Brasil
}

\section{Geostatistics as a tool to estimate rainfall variability in Pernambuco, Brazil}

\author{
Timóteo Herculino da Silva Barros \\ Escola superior de Agricultura Luiz de Queiroz \\ E-mail: timoteo@usp.br \\ OrclD: https://orcid.org/0000-0002-1242-9889
}

Fabiani Denise Bender Centro Nacional de Monitoramento e Alertas de Desastres Naturais

E-mail: fabianidenise@gmail.com

OrcID: https://orcid.org/0000-0003-3634-5265

Flávia Rosana Barros Silva Universidade Federal Rural de Pernambuco

E-mail: barrosflaviar@gmail.com OrclD: https://orcid.org/0000-0003-4386-061X

Jefferson Viera José Universidade Federal do Acre

E-mail: jfvieira@usp.br

OrcID: http://orcid.org/0000-0003-1384-0888

Jéfferson de Oliveira Costa Instituto Federal do Norte de Minas Gerais

E-mail: costajo@usp.br

OrclD: https://orcid.org/0000-0002-5387-7880

Rubens Duarte Coelho Escola superior de Agricultura Luiz de Queiroz

E-mail: rdcoelho@usp.br

OrcID: https://orcid.org/0000-0002-0472-8301

Resumo: O conhecimento do comportamento espaço temporal da precipitação pluviométrica nesse contexto apresenta um papel relevante quando o objetivo é tomar decisões a respeito do recurso mais importante. $O$ objetivo deste trabalho foi investigar a contribuição da longitude, da latitude e a covariável altitude, como variáveis auxiliares na obtenção de estimativas da distribuição espacial da precipitação anual média no estado de Pernambuco. Dentre os modelos testados o exponencial apresentou melhor ajuste aos dados observados, e as tendências apresentaram forte dependência espacial e estão diretamente correlacionadas com a precipitação pluviométrica média anual. A utilização da ferramenta apresentou-se eficaz na estimativa da precipitação pluviométrica, e pode ser empregada em diversas áreas do conhecimento, principalmente como ferramenta de apoio para tomada decisão.

Palavras-chave: Distribuição da precipitação pluviométrica; Semivariograma; Semiárido nordestino.

Abstract: The knowledge of the temporal space behavior of the rains in this context plays an important role when the objective is to make decisions about the most important resource. The objective of this work was to investigate the contribution of longitude, latitude and the covariable altitude, as auxiliary variables in obtaining estimates of the spatial distribution of average annual precipitation in the state of Pernambuco. Among the models tested, the exponential presented a better fit to the observed data, and the trends showed a strong 
spatial dependence and are directly correlated with the average annual rainfall. The use of the tool proved to be effective in estimating rainfall, and can be used in several areas of knowledge, mainly as a support tool for decision making.

Keywords: Rainfall distribution; Semivariogram; Northeastern semiarid.

Data de recebimento: $20 / 05 / 2020$

Data de aprovação: 02/08/2020

DOI: https://doi.org/10.30612/agrarian.v13i50.11982

\section{Introdução}

A precipitação pluviométrica apresenta-se como a variável meteorológica mais importante do ciclo hidrológico, pois o seu regime influencia diretamente em várias atividades, entre elas: a agricultura, a pesca, a pecuária e, principalmente, o abastecimento de água para o consumo humano que vem sendo a preocupação em todo o estado de Pernambuco (QUEIROZ et al., 2020).

O regime hídrico no nordeste brasileiro apresenta grande variabilidade na intensidade das precipitações pluviométricas assim como na sua distribuição espaço-temporal. A Região Nordeste do Brasil apresenta uma situação vulnerável a que está exposta a maior parte do seu território em decorrência de um regime de precipitação pluviométrica bastante irregular, que apresenta grandes riscos para os sistemas de agricultura no qual não detém de técnicas de irrigação e do próprio recurso para minimizar as perdas e os baixos índices de produtividade provocados pela redução dos regimes hídricos na região. Outros fatores, tais como uma baixa capacidade de retenção de umidade no solo e altas taxas de evaporação e evapotranspiração, contribuem para agravar o problema (YONTS et al., 2018).

O conhecimento da distribuição da precipitação pluviométrica nesse contexto apresenta um papel relevante quando o objetivo é tomar decisões a respeito do recurso mais importante do planeta (BAO et al., 2012) e não diferente, para o planejamento agrícola adequado. No entanto, longas séries de dados observados desta variável são muitas vezes escassos, devido à baixa densidade de distribuição de pluviômetros ou mesmo em decorrência de falhas nas medidas (BENDER \& SENTELHAS, 2018; BATTISTI et al., 2019). Por isso, métodos de estimativas, tem se mostrados eficientes em regiões que sofrem com a ausência de séries de dados observados.

A geoestatística trata-se de uma ferramenta muito poderosa, que vem sendo usada em diversas áreas do conhecimento, atualmente utilizada para fazer estimativas da variabilidade espacial da precipitação pluviométrica de um município, uma bacia hidrográfica ou até mesmo um estado (BUTTAFUOCO et al., 2017; CASTRIGNANÒ et al., 2018). Os dados de precipitação pluviométrica de uma determinada região normalmente são obtidos em postos de coletas pontuais, sendo assim não é possível saber calcular o quando pode chover uma região vizinha, o que por sua vez gera a necessidade de utilização de métodos de interpolação espacial para o seu mapeamento, entre os quais se destaca a krigagem (MUÑOZ-NÁJERA et al., 2020).No entanto, a Krigagem apresenta-se como uma das ferramentas dentro da geoestatística que possibilita a visualização do comportamento de uma determinada variável que no caso desse estudo é a precipitação pluviométrica (CORTÉS et al., 2017; MUÑOZ-NÁJERA et al., 2020).

Outros avanços referentes ao uso da geoestatística vêm sendo empregados com o intuído de analisar a variabilidade espacial e temporal que interferem diretamente o rendimento das culturas agrícolas em geral (CASTRIGNANÒ et al., 2018). A ferramenta nesse contexto possibilita maximização da energia empregada para determinados processos e minimização dos recursos financeiros destinados às atividades. Várias áreas da ciência vêm utilizando a geoestatística como ferramenta para facilitar a tomada de decisão (BUTTAFUOCO et al., 2017).

O trabalho tem como hipótese a possibilidade do zoneamento da distribuição da precipitação pluviométrica por meio do uso da geoestatística, assim o objetivo foi estudar por meio da geoestatística, a variabilidade espacial do regime da precipitação pluviométrica no estado de Pernambuco, Brasil, e assim avaliar seu comportamento da distribuição no estado.

\section{Material e Métodos}




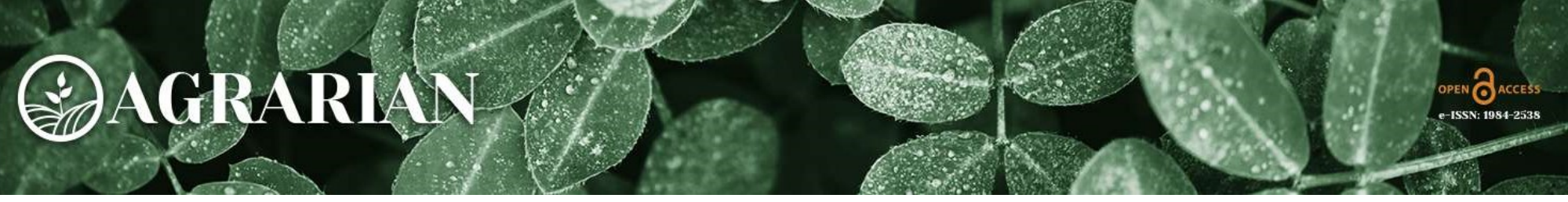

A região de estudo compreende o estado de Pernambuco, situado a centro-leste da região Nordeste, com uma área de $98.281 \mathrm{~km}^{2}$. Limita-se, ao Norte, com os estados do Ceará e a Paraíba; a Oeste, com o Piauí; ao Sul, com Bahia e Alagoas; a Leste, com o Oceano Atlântico banhando seu litoral. Tem 185 municípios, divididos em três grandes regiões geoeconômicas: Litoral/Mata, Agreste e Sertão/São Francisco.

As séries de dados mensais de precipitação pluvial, utilizadas neste estudo, foram obtidas da base de dados da Agência Nacional de Águas (ANA, 2020). Consideraram-se 31 anos de dados, obtidos dos registros do período histórico de 1983 a 2013, em 150 postos pluviométricos espalhados em todo o estado.

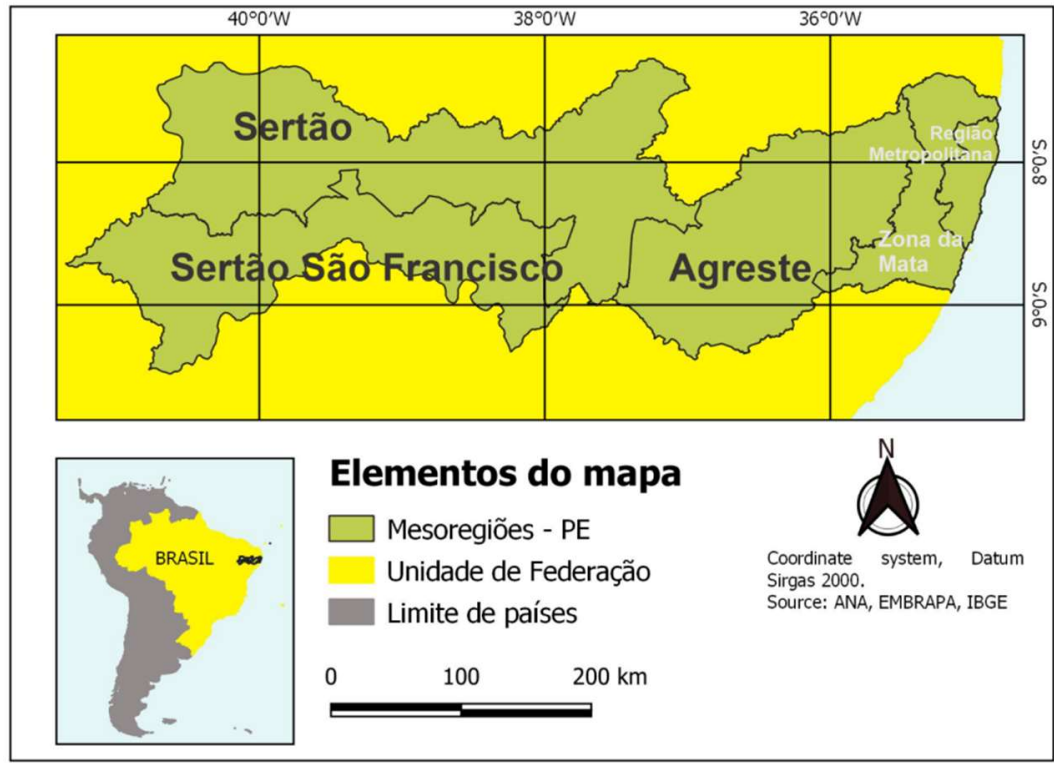

Figura 1. Localização da região de estudo que compreende o estado de Pernambuco, Brasil.

O pacote geoR (RIBEIRO JUNIOR \& DIGGLE, 2001), do programa R (R DEVELOPMENT CORE TEAM, VIENA, AT) foi utilizado para verificação de dependência espacial por meio de análise de semivariogramas da precipitação utilizando envelope de simulação do pacote de análise. Para observar se os dados brutos apresentavam normalidade ou não, foi aplicado o teste de Shapiro e Wilk, (1965). Para identificar a dependência espacial, foram usados diferentes modelos de semivariogramas teóricos: de correlação esférico, exponencial, gaussiano, circular e cúbico para determinar o modelo mais adequado.

$\mathrm{Na}$ comparação dos modelos com diferentes estruturas espaciais e funções de correlação, utilizou-se o critério de Akaike - AIC, calculado pelo dobro da diferença do logaritmo natural da função de máxima verossimilhança e o número de parâmetros do modelo, sendo selecionado o modelo que apresentou menor valor de AIC, Critério Bayesiano de Schwarz - BIC, O Critério Bayesiano de Schwarz (BIC) tem como pressuposto a existência de um "modelo verdadeiro" que descreve a relação entre a variável dependente e as diversas variáveis explanatórias entre os diversos modelos sob seleção. Já para determinação dos mapas de interpolação, eles foram gerados com a utilização do pacote geoR (RIBEIRO JR. \& DIGGLE, 2001), usando a técnica de Krigagem ordinária.

\section{Resultados e Discussão}

A transformação dos dados de precipitação do estado de Pernambuco, e determinação do valor lambda $(-0,87) \lambda$ Figura $2(A)$, no qual se verifica que após a transformação Box-Cox ampliado apresentou assimetria (B). Após aplicarmos essa transformação aos dados, as especificações e os parâmetros do processo como média, variabilidade inerente e total são alcançados para os dados transformados, aplicando a análise via dados normais. Da mesma forma, os índices são calculados para os dados transformados com a distribuição normal. 

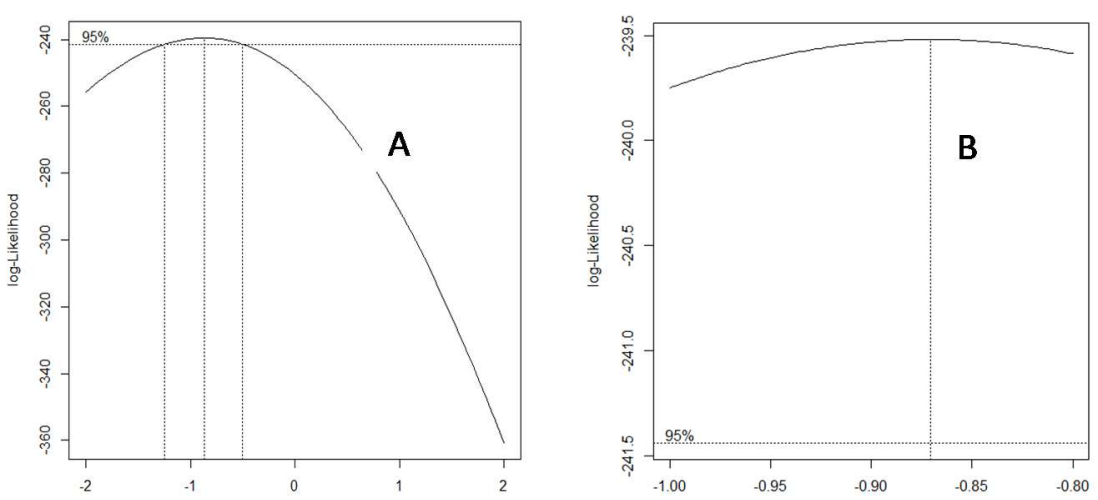

Figura 2. Transformação dos dados de precipitação pluviométrica do estado de Pernambuco, e determinação do valor lambda $-\boldsymbol{\Lambda}(\mathrm{A})$ e, ampliação do gráfico para melhor observação (B).

A análise exploratória e padrão espacial dos dados de precipitação anual considerando tendências espaciais estão apresentadas na Figura $3(A)$, já sem tendência espacial dos dados tanto nas coordenadas " $x$ " (B) quanto nas coordenadas " $y$ " (C) e o histograma da variável de precipitação (D).
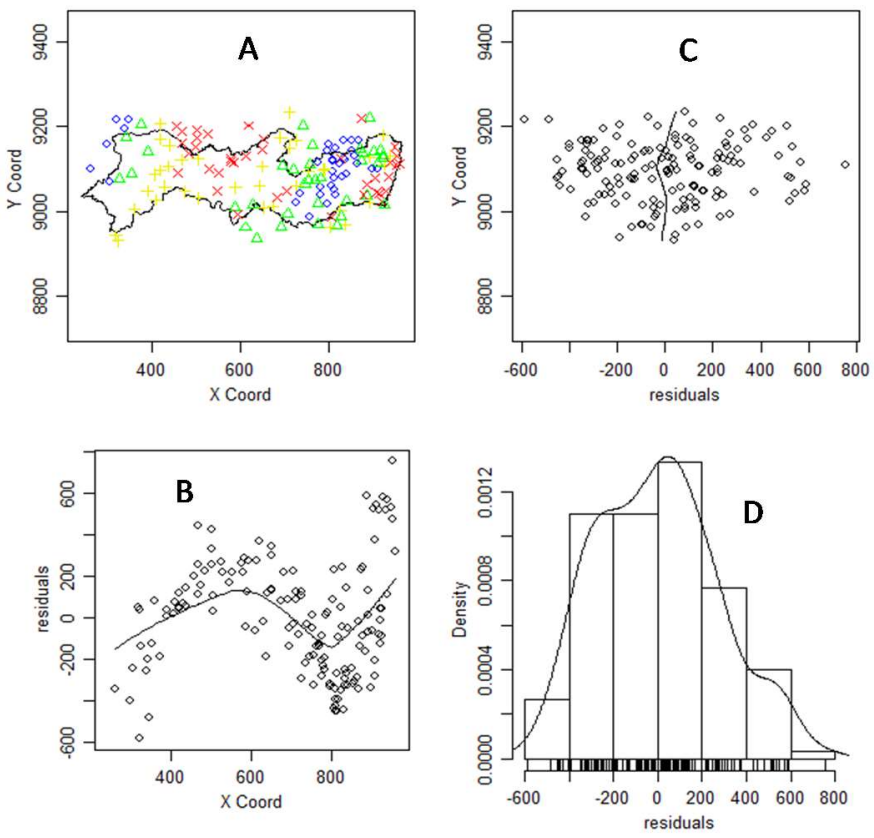

Figura 3. Análise exploratória para os dados transformados, referente aos dados históricos pluviométricos do estado de Pernambuco, para o período de 1983-2013.

A forma de observação de analisar se existe dependência espacial ocorreram por meio de simulações com os dados da precipitação para no estado de Pernambuco (Figura 4). Observa-se a existência de dependência espacial no semivariograma é a identificação de pontos na área externa do envelope de simulação Figura $4(\mathrm{~B})$, esses pontos indicam dependência espacial dos valores no estudo. Como podemos observar há vários pontos fora da linha de confiança Figura 4 (B). Existem alguns pontos fora do envelope o que indica indício de que há continuidade espacial. 

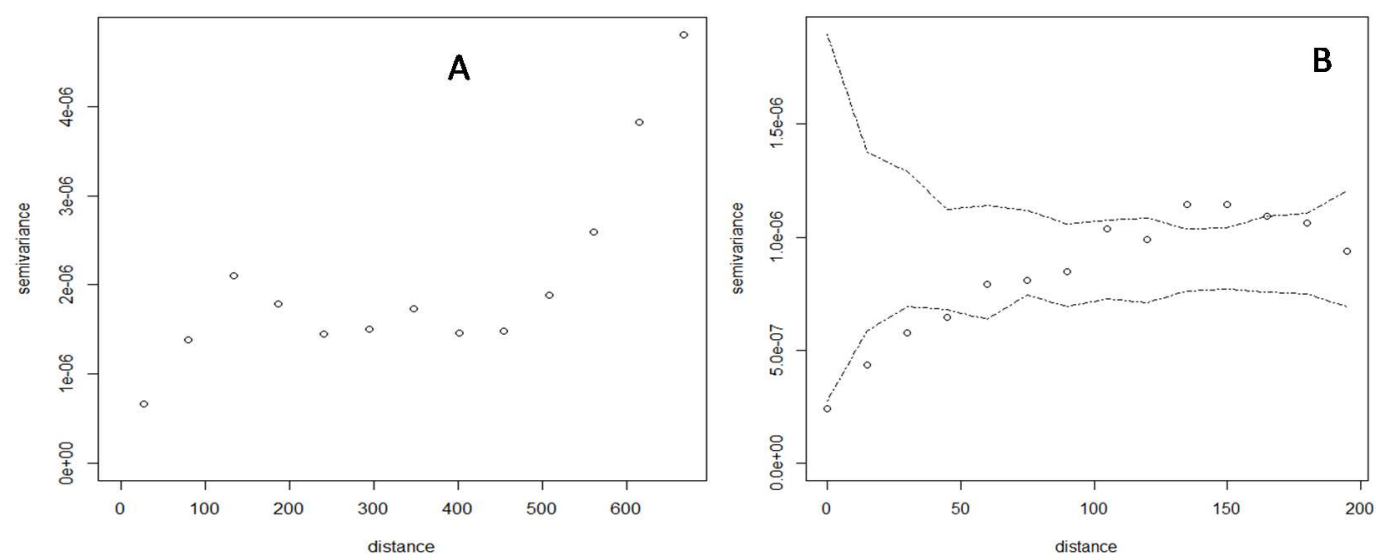

Figura 4. Semivariograma referente aos dados de precipitação pluviométrica anual media no estado de Pernambuco (A), e semivariograma envelopado (B).

Os valores das estimativas dos modelos utilizados, assim como os valores de respostas dos testes critério de Akaike - AIC e Critério Bayesiano de Schwarz - BIC para escolha do melhor modelo são apresentados na Tabela 1. Por meio de funções de correlação o modelo que melhor se ajustou foi o exponencial, apresentando o menor valor de AIC (2019.97), ao passo que os modelos cuibic e gaussiano apresentaram os piores ajustes. Para o metodo de analise do BIC o modelo exponencial também apresentou o melhor comportamento, sendo assim escolhido no presente estudo. Resultado semelhante também foi encontrado em Minas Gerais com dependência espacial de precipitações pluvial intensas (MELLO \& VIOLA, 2013).

Vale salientar que ao utilizar o AIC admite-se que dentre os modelos avaliados nenhum é considerado o que realmente descreve a relação entre a variável dependente e as variáveis exploratórias ou o "modelo verdadeiro" e então, tenta-se escolher o modelo que minimize a divergência. Com o BIC, está implícito que existe o modelo que descreve a relação entre as variáveis envolvidas e o critério tenta maximizar a probabilidade de escolha do verdadeiro modelo (BOZDOGAN, 1987; DEXTER et al., 2008).

Tabela 1. Estimativa dos modelos de maxima verossimilhança, associados aos modelos, e os indíces testes Critério de Akaike (AIC) e Critério Bayesiano de Schwarz (BIC).

\begin{tabular}{lccccc}
\hline \multirow{2}{*}{ Modelos } & Patamar & Alcance & Efeito Pepita & & BIC \\
\cline { 2 - 5 } & Tausq & Sgmasq & Phi & AlC & 2038 \\
\hline Exponencial & 4553,25 & 87058,48 & 64,26 & 2019,97 & 2221,72 \\
Gaussiano & 27945,15 & 57863,05 & 78,23 & 2203,66 & 2040,26 \\
Circular & 18155,34 & 66738,08 & 138,19 & 2022,19 & 2040,23 \\
Esférico & 15953,51 & 69026,14 & 152,83 & 2022,17 & 221,72 \\
Cúbico & 27458,23 & 57744,9 & 185,16 & 2203,66 & 220 \\
\hline
\end{tabular}

Na Figura 5 observa-se os valores preditos de precipitação pluviométrica nas simulações para os dados da normal climatológica do estado de Pernambuco. A Figura mostra o padrão da distribuição espacial da precipitação pluviométrica em termos de quantidade anual em milímetros, onde a cor azul indica as maiores médias de precipitação, $2000 \mathrm{~mm}^{2} \mathrm{ano}^{-1}$, e as cores laranja e vermelha os menores volumes de precipitação pluviométrica acumulada anualmente, próximo a $400 \mathrm{~mm}$ ano-1. Os maiores valores simulados para a região costeira do estado podem ser explicados, pela influência que a região sofre do Oceano Atlântico, enquanto as cores laranja e vermelha indicam menores média de precipitação pluviométrica. Vale ressaltar que o extremo leste do estado apresentou as menores precipitações médias, chegando a valores inferiores a $400 \mathrm{~mm}^{\mathrm{ano}}{ }^{-1}$ (SÁNCHEZ et al., 2015)

As precipitações pluviométricas mensais analisadas por Nóbrega et al. (2018), corroboram com os valores estimados no presente estudo, de acordo com a Figura 4, com os maiores volumes nas cidades próximas ao litoral, nas cidades de Recife e Rio Formoso, enquanto as cidades do Agreste e Sertão apresentam distribuições próximas. O estado de Pernambuco apresenta variação na distribuição espacial das precipitações 
pluviométricas muito abrupta, onde cada região demonstra suas peculiaridades. De acordo com Ravellys et al., (2019) esse tipo de análise permite detectar na mesorregião da Região Metropolitana certa regularidade de distribuição para os episódios chuvosos e secos, enquanto para as localidades do Agreste de Pernambucano a predominância de episódios extremamente secos.

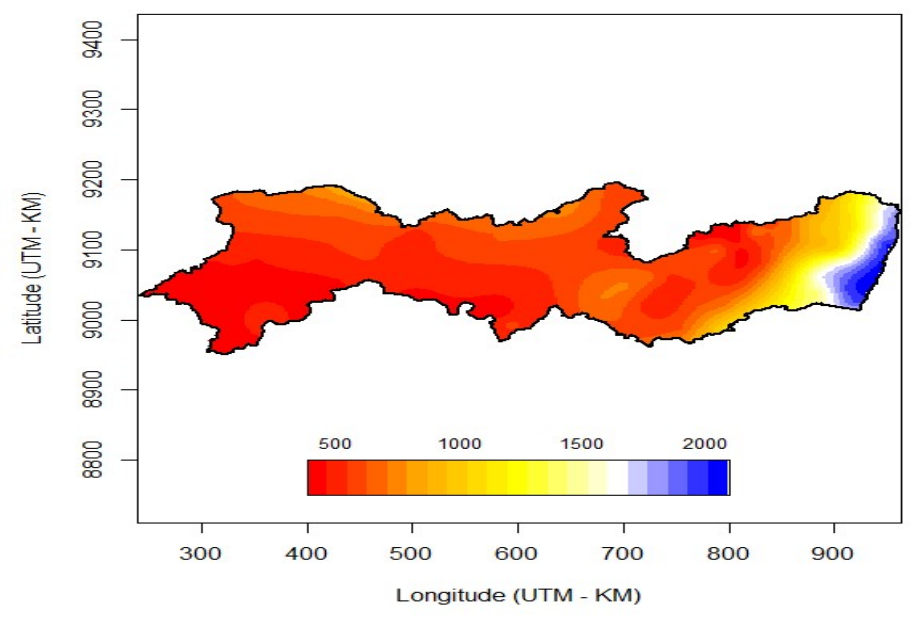

Figura 5. Totais de precipitação pluviométrica média anual $(\mathrm{mm})$, para a região do estado de Pernambuco, obtida por intermédio do método de interpolação linear krigagem ordinário dos valores preditos.

No Litoral/Zona da Mata, o clima é tropical úmido, com precipitação pluviométrica de outono a inverno, numa média histórica situada entre 1.500 a 2.000 mm anuais; na área de Agreste e Sertão, (que corresponde a dois terços do território estadual) predomina o clima tropical semiárido, com baixa pluviosidade (média de 600 $\mathrm{mm}$ anuais de precipitação pluviométrica) concentrada no verão para o Sertão, e de maio a julho para a região do agreste, mas sujeita a irregularidades que acabam provocando secas prolongadas. $\mathrm{Na}$ faixa de transição entre a Zona da Mata e o Sertão, a pluviosidade fica entre 650 a $1.000 \mathrm{~mm}$ anuais (HOUNSOU-GBO et al., 2016).

Pernambuco tem um total de $98.281 \mathrm{~km}^{2}$ dos quais $87.317 \mathrm{~km}^{2}$ (que é a soma das regiões Sertão e Agreste) localizados no chamado Polígono das Secas. Essa área corresponde a $89 \%$ do território pernambucano. A parte mais crítica é a região Oeste do Estado (onde estão municípios como Afrânio, Santa Filomena, Terra Nova e Floresta), que tem as menores e mais irregulares precipitações pluviométricas: a média anual não supera os 500 milímetros, com o registro de um elevado número de anos em que as precipitação pluviométrica não alcançam os $200 \mathrm{~mm}$ anuais.

A Figura 6 apresenta o mapa de erros padrão de predição e a variância de krigagem referente aos totais de precipitação pluviométrica média anual da normal climatológica estimados por intermédio do interpolador linear de krigagem ordinário, para a região do estado de Pernambuco.




Figura 6. Mapa de erro padrão de predição (incerteza de predição), ou seja, variância de krigagem referente aos totais de precipitação pluviométrica média anual da normal climatológica, para a região do estado de Pernambuco, estimados por intermédio do método de interpolação linear krigagem ordinário dos valores preditos.

Os pontos mais próximos, ou seja, a coloração mais próxima da tonalidade de vermelho indica que a possibilidade de erros com a simulação é menor, e assim apresenta maior nível de confiabilidade nas predições, enquanto as tonalidades mais próximas ao verde são as que apresentam as maiores incertezas nas simulações. De forma geral as regiões da Zona da Mata e Agreste do estado, são as que apresentam o maior número de pontos de observações, garantindo assim uma melhor confiabilidade na malha de valores estimados.

\section{Conclusões}

A utilização da ferramenta apresenta-se muito útil no que se refere a análise de dados, e pode ser empregada em diversas áreas do conhecimento, principalmente como ferramenta para tomada decisão. Há a possibilidade de uso de ferramentas de geoestatística para simular dados de precipitação pluviométrica em locais nos quais não se tem estações pluviométricas.

Vale destacar que o método que apresentou melhor desempenho nas estimativas foi o modelo Exponencial, e que a densidade de distribuição dos dados observados, apresenta influência no desempenho das estimativas, isto é, quanto maior a densidade de dados, menor o erro associado aos valores preditos.

\section{Referências}

ANA. ANA discute recursos hídricos do Nordeste em Simpósio - Agência Nacional de Águas.

Disponível em: <https://www.ana.gov.br/noticias-antigas/ana-discute-recursos-hadricos-do-nordeste-em.201903-15.2688459806>. Acesso em: 13 maio. 2020.

BAO, Z.; ZHANG, J.; LIU, J.; WANG, G.; YAN, X.; WANG, X.; ZHANG, L.I. Sensitivity of hydrological variables to climate change in the Haihe River basin, China. Hydrological Processes, v. 26, n. 15, p. 2294-2306, 15 jul. 2012.

BATTISTI, R.; BENDER, F. D.; SENTELHAS, P. C. Assessment of different gridded weather data for soybean yield simulations in Brazil. Theoretical and Applied Climatology, v. 135, n. 1-2, p. 237-247, 2019.

BENDER, F. D.; SENTELHAS, P. C. Solar radiation models and gridded databases to fill gaps in weather series and to project climate change in Brazil. Advances in Meteorology, v. 2018, 2018.

BOZDOGAN, H. Model selection and Akaike's Information Criterion (AIC): The general theory and its analytical extensions. Psychometrika, v. 52, n. 3, p. 345-370, set. 1987.

BUTTAFUOCO, G.; CASTRIGNANÒ, A.; CUCCI, G.; LACOLLA, G.; LUCÀ, F. Geostatistical modelling of within-field soil and yield variability for management zones delineation: a case study in a durum wheat field. Precision Agriculture, v. 18, n. 1, p. 37-58, 1 fev. 2017.

CASTRIGNANÒ, A.; BUTTAFUOCO, G.; QUARTO, R.; PARISI, D.; VISCARRA ROSSEL, R.A.; TERRIBILE, F., LANGELLA, G., VENEZIA, A. A geostatistical sensor data fusion approach for delineating homogeneous management zones in Precision Agriculture. Catena, v. 167, p. 293-304, 1 ago. 2018.

CORTÉS, J.L.; BAUTISTA, F.; DELGADO, C.; QUINTANA, P.; AGUILAR, D.; GARCÍA, A.; FIGUEROA, C.; GOGICHAISHVILI, A. Distribución espacial de los metales pesados en polvos urbanos de la ciudad de Ensenada, Baja California, México. Revista Chapingo, Serie Ciencias Forestales y del Ambiente, v. 23, n. 1, p. 47-60, 1 jan. 2017

DEXTER, A.R.; CZYZ, E.A.; RICHARD, G.; RESZKOWSKA, A. A user-friendly water retention function that takes account of the textural and structural pore spaces in soil. Geoderma, v. 143, n. 3-4, p. 243-253, 2008.

HOUNSOU-GBO, G.A.; SERVAIN, J.; ARAUJO, M.; MARTINS, E.S.; BOURLÈS, B.; CANIAUX, G.. Oceanic Indices for Forecasting Seasonal Rainfall over the Northern Part of Brazilian Northeast. American Journal of 
Climate Change, v. 05, n. 02, p. 261-274, 31 maio 2016.

MELLO, C. R.; VIOLA, M. R. Mapeamento de chuvas intensas no estado de Minas Gerais. Revista Brasileira de Ciencia do Solo, v. 37, n. 1, p. 37-44, jan. 2013.

MUÑOZ-NÁJERA, M.A.; TAPIA-SILVA, F.O.; BARRERA-ESCORCIA, G.; RAMÍREZ-ROMERO, P. Statistical and geostatistical spatial and temporal variability of physico-chemical parameters, nutrients, and contaminants in the Tenango Dam, Puebla, Mexico. Journal of Geochemical Exploration, v. 209, p. 106435, 1 fev. 2020.

NÓBREGA, R.L.B.; GUZHA, A.C.; LAMPARTER, G.; AMORIM, R.S.S.; COUTO, E.G.; HUGHES, H.J.; JUNGKUNST, H.F.; GEROLD, G. Impacts of land-use and land-cover change on stream hydrochemistry in the Cerrado and Amazon biomes. Science of the Total Environment, v. 635, p. 259-274, 2018.

QUEIROZ, M.G.; SILVA, T.G.F.; ZOLNIER, S.; SOUZA, C.A.A.; DE SOUZA, L.S.B.; NASCIMENTO ARAÚJO, G.; JARDIM, A.M. R.F.; MOURA, M.S.B. Partitioning of rainfall in a seasonal dry tropical forest. Ecohydrology and Hydrobiology, 8 fev. 2020.

RAVELLYS, L.; ALCÂNTARA, P. D.; ALVES, L.; RAYANE, I.; COSTA, D.A. Journal of Environmental mesorregiões do estado de Pernambuco Evaluation of probabilistic models for heavy rains in the mesoregions of the state of Pernambuco. v. 01, p. 90-103, 2019.

RIBEIRO JR., P. J.; DIGGLE, P. J. geoR: A package for geostatistical analysis. R-News, 2001.

SÁNCHEZ, E.; SOLMAN, S.; REMEDIO, A.R.C.; BERBERY, H.; SAMUELSSON, P.; DA ROCHA, R.P.; MOURÃO, C.; LI, L.; MARENGO, J.; CASTRO, M.; JACOB, D. Regional climate modelling in CLARIS-LPB: a concerted approach towards twentyfirst century projections of regional temperature and precipitation over South America. Climate Dynamics, v. 45, n. 7-8, p. 2193-2212, 1 out. 2015.

SHAPIRO, S. S.; WILK, M. B. An Analysis of Variance Test for Normality (Complete Samples). Biometrika, v. 52, n. 3/4, p. 591, 1965. Disponível em: <http://www.jstor.org/stable/2333709?origin=crossref>.

YONTS, C.D.; HAGHVERDI, A.; REICHERT, D.L.; IRMAK, S. Deficit irrigation and surface residue cover effects on dry bean yield, in-season soil water content and irrigation water use efficiency in western Nebraska high plains. Agricultural Water Management, v. 199, p. 138-147, 1 fev. 2018. Disponível em:

<https://www.sciencedirect.com/science/article/pii/S037837741730416X>. Acesso em: 12 mar. 2019. 